\title{
Gluten sensitivity: real or not?
}

\author{
Fiona Godlee editor, BMJ
}

Does non-coeliac gluten sensitivity exist? The author of this week's Patient Journey has no doubt of it (doi:10.1136/bmj. e7982). After years of unexplained ill health, a chance conversation on an internet forum led him to try an exclusion diet. The results were dramatic, he says. "Within a week of excluding gluten and lactose from my diet, all my symptoms had dramatically improved in just the same way as when I previously starved myself." Accidental rechallenge brought the symptoms back "within hours."

Despite symptomatic relief he wanted a "proper diagnosis." He saw Kamran Rostami, whose account of the condition accompanies the patient's story. "Patients have negative immunoallergy tests to wheat and negative coeliac serology; normal endoscopy and biopsy; symptoms that can overlap with coeliac disease, irritable bowel syndrome, and wheat allergy." Symptoms resolve on a gluten-free diet. Since there are no biomarkers, gluten sensitivity is the ultimate diagnosis of exclusion.

This, and the lack of a disease mechanism, may explain why not everyone is convinced. Luca Elli would like to see aspects of gluten sensitivity clarified before we start "treating" people for this new "disease" (doi:10.1136/bmj.e7360). He argues that although exclusion diets aim to improve patients' quality of life, they also in themselves reduce quality of life, and we don't know about possible complications. And what of the financial incentives for companies promoting gluten-free products? The worldwide market is now estimated to be worth up to $\$ 850 \mathrm{~m}$ a month. Is gluten sensitivity different from irritable bowel syndrome, asks Elli, or is it simply a variant that benefits from a common therapeutic approach?
Imran Aziz and colleagues look to the published literature to resolve these uncertainties (doi:10.1136/bmj.e7907). Compared with coeliac disease, gluten sensitivity has been little researched, but a few randomised trials suggest that this is a real condition, affecting $6 \%$ of nearly 6000 people tested in a Maryland clinic. The worldwide shift to the Mediterranean diet may explain the rising prevalence, they say. A multicentre trial is currently recruiting people with gluten sensitivity for challenge with gluten or placebo. Meanwhile, these authors advise that patients in whom coeliac disease has been excluded through serology tests and duodenal biopsy should be told that their problem is a newly recognised clinical entity for which we do not yet fully understand the natural course or pathophysiology.

This advice will contribute further to the rising number of people being tested for coeliac disease. In a letter published this week, David Unsworth and colleagues report on the "explosion of requests" for serological testing since 2007, particularly from primary care (doi:10.1136/bmj.e8120). NICE guidance in 2009 did little to check the rise, they say. And as the number of people being tested has risen, the proportion of those in whom coeliac disease is confirmed has fallen to just over $1 \%$, no better than would be achieved by random screening. They call for more targeted testing, limited to groups in whom detection rates are highest: children with failure to thrive, family history, or type 1 diabetes, and adults attending diabetes and gastroenterology clinics. This makes sense, except that detection rates are not the only way to judge the value of a test. As our Patient Journey shows, a negative test result can be just as valuable to the patient.

Cite this as: BMJ 2012;345:e8450

๑ BMJ Publishing Group Ltd 2012 